\title{
Complementary and Alternative Medicines (Cam) Use among Cancer Patients: An Overview and the Decision Making
}

\section{Maryam Farooqui*}

Department of Pharmacy Practice, Unaizah College of Pharmacy, Unaizah College of Pharmacy, Qassim University, Qassim, Saudi Arabia

*Corresponding author: Maryam Farooqui, Department of Pharmacy Practice, Unaizah College of Pharmacy, Unaizah College of Pharmacy, Qassim University, PO BOX 5888 Unaizah 51911, Qassim, Saudi Arabia, E-mail: maryamfarooqui.uitm@gmail.com

Received date: October 17, 2017, Accepted date: October 22, 2017, Published date: October 28, 2017

Copyright: @ 2017 Farooqui M. This is an open-access article distributed under the terms of the Creative Commons Attribution License, which permits unrestricted use, distribution, and reproduction in any medium, provided the original author and source are credited.

\begin{abstract}
Caner is a leading cause of death worldwide which requires a multidisciplinary treatment approach. Safety and efficacy of many of the modern therapies have been well established but still use of complementary and alternative medicines (CAM) are on rise among cancer patients. CAM decision making process is complex which is affected by many social and disease related variables. Prevalence based studies regarding CAM use reported a wide range of variation in percentage use of CAM among cancer patients which could be due to the way different studies defined CAM in their studies. Understanding CAM use decision making is crucial as it is highly individualized and revolves around several issues which can benefit patients and their care givers in treatment success.
\end{abstract}

Keywords: Complementary and alternative medicine; Cancer; Healing; WHO

\section{Introduction}

Cancer related deaths are expected to rise to over 13.1 million in 2030 (WHO) [1]. Treatments for cancer are multidisciplinary which requires the skills of a team of experts. Conventional treatments are those that are extensively merged into the modern health care system. They are usually considered as Evidence Based Medicines (EBM) i.e. the safety and efficacy of these therapies have been well established through clinical trials.

The use of CAM for health and for healing purposes has long existed in human society. In the modern era, therapies which are now used to complement or as an alternative to conventional medicines, were the only way of cure in ancient times.

However, a boost in modern drug delivery options excluded CAM. The bases of many of the modern treatments for cancer originated in pre-modern society and were used for treatment and prevention.

For example, Vincristine (Oncovin ${ }^{\circ}$ ) was used as folk remedy for centuries until scientists revealed that this medicinal plant has a number of alkaloids, some of which are responsible for myelosuppression (decreased activity of the bone marrow [2].

In the modern era of the $21^{\text {st }}$ century, many of the underdeveloped countries such as African and some of the south East Asian countries rely solely on CAM as a source of treatment [3]. Lack of health care facilities, poverty and the disease burden on the health care system forced many patients to choose CAM as their first option for treatment.

At the same time cultural influences and a lack of knowledge regarding modern therapies turns many cancer patients towards CAM. In this context defining CAM is a difficult task, as what is considered as $\mathrm{CAM}$ in one region of the world could be part of conventional treatment in other regions.

\section{CAM Definition}

In recent years' significant emphasis has been given to defining CAM from a cultural context, since what is considered as CAM in European/Western cultures could be part of daily health care practice in other regions of the world (for e.g. acupuncture in North America vs. China). A blended and more flexible definition of CAM has been given by Zollman and Vickers who define CAM as "a broad domain of healing resources that encompasses all health systems, modalities and practices, and their accompanied theories and beliefs other than those intrinsic to the politically dominated health system of a particular society or culture in a given historical period" [4]. A similar definition is given by the WHO, where CAM is defined as "a broad set of health care practices that are not part of that country's own tradition and are not integrated into the dominant health care system" [5]. Within this thesis, CAM is defined as "a group of diverse medical and healthcare systems, practices, and products that are not currently part of conventional medicines" [6]. This article provides different types of CAM therapies classified by the US National Centre for Complementary and Alternative Medicines (NCCAM).

\section{Prevalence of CAM use}

In general CAM use is common among healthy individuals as well as patients with chronic diseases such as arthritis, HIV/AIDS and cardiovascular diseases [7-9]. On average $40 \%$ of cancer patients from western societies including Australia, Canada, Europe, New Zealand and the USA use CAM [10]. In the US, a population based survey reported that $40 \%$ of cancer survivors had used CAM during the previous year [11]. In a systemic review of 52 studies including 14 countries from western and eastern regions showed that the prevalence of CAM use ranged from as low as $5 \%$ to as high as $60 \%$ with an average prevalence of $31.4 \%$ in adult cancer patients [12].

Very few studies reported that the prevalence of CAM use among Asian cancer patients which is relatively higher than that of Western countries [13]. For example, among the Asian cancer population the reported CAM prevalence is, China (93.4\%), Sri Lanka (67.4\%), 
Malaysia (64\%), Thailand (60.9\%), Singapore (56\%), Pakistan (54.5\%), Turkey (42.3\%) and in India (34.3\%) [14-20]. The wide variation in the frequency of CAM use shown within these studies could be due to understandings of CAM, the list of CAM therapies provided during interviews (predefined list of CAM given vs. recall of CAM without being prompted), frequency of CAM use and restrictions to consider only certain CAM therapies as a study's objective.

The understanding of the concept of CAM may vary the pattern of response. Different people consider CAM in different ways, for example, prayers and spiritual therapies are perceived as a daily life practice for some patients and thus not reported as CAM but for others it can be considered as CAM [21]. It is also noticeable that the duration of CAM use may also affect the percentage reported. Surveys investigating CAM use since cancer diagnosis may have a high CAM prevalence compared to surveys investigating CAM use within a limited duration (for example, the last six months or a year). Similarly, one may also argue that face to face interviews may introduce some bias when eliciting responses, especially if the survey is conducted in a conventional oncology setting or the interviewer is a nurse or pharmacist from the same setting thus forcing patients to respond positively in a socially desirable manner. Since fear of termination of therapy is reported among the reasons for CAM non-disclosure this debate could be acceptable to some extent [22].

\section{CAM Use Decision}

The decision to use CAM is a highly complex process which revolves around many issues. An individual's perspective towards health and disease may affect the treatment decision. Factors affecting CAM use are diverse and are not merely due to a dissatisfaction with conventional medicines but more as an alternative to avoid cancer recurrence, to strengthen the body after or during cancer treatment and for psychological wellbeing [23]. Mao and colleagues while investigating CAM users' unmet need, found out that emotional, physical, nutritional, financial, treatment related information and employment factors were the common unmet needs among $50 \%$ of cancer patients [24]. A similar review concluded that the CAM use was associated with a strong belief in CAM, a need for control and considering CAM as a last resort and hope for cure [25].

The emotional impact of cancer diagnosis, perceptions of the disease, the availability of different treatment options and the cost of therapy may act as pulling or pushing factors in CAM use [25]. Patients with a passive behaviour towards treatment may rely on their physician's advice. However, patients actively participating in the treatment decision may contribute to the CAM decision making process. The first phase in the CAM decision making process involves gathering information about CAM. Due to the availability of a wide range of CAM, patients usually find themselves with a pool of CAM therapies where choosing one or picking the best one is always a challenging task [26]. Recommendations from friends and family members and other cancer survivors are some of the sources reported to help patients in CAM selection [27,28]. Patient judgment to the potential risks and benefits of CAM and the possible interactions with conventional medicines may also affect CAM decision [25].

The cost and availability of CAM may also affect the decision to use CAM [29,30]. Although low cost and easy access are among the reasons given for CAM use this is not true in all cases [31]. Homemade traditional therapies may provide patients with easy and cheap access to these therapies, but most of the commercial products including mega vitamins, herbal drinks and supplements are relatively expensive. Traditional Chinese Medicines (TCM) is easily available in China but may not be so easily available for patients from other parts of the world. Travelling or shipment cost may also affect a patient's decision to use CAM. Diagnosis with cancer may also affect a patient's productivity (job loss) or the cost of conventional treatment may be a burden [32]. In such situations the extra cost of CAM therapies may affect patient decisions regarding using CAM. Thus beside affordability, the availability of certain CAM may affect the CAM decision making process [33]

Ascertaining the appropriate phase of cancer to start a CAM in order to avoid interaction with conventional therapies is another aspect which affects the decision of CAM use [34]. Using one CAM at a time to avoid CAM-CAM interactions as well as to evaluate the effectiveness of a certain CAM could also be an important factor in CAM decisions. Similarly, patient decisions to use non-invasive CAM therapies while undergoing conventional treatment may also affect the choice of CAM modalities. Therapies like massage, relaxation techniques and yoga, Qigong, and music therapies have been commonly used by cancer patients to meet those psychological needs which are not fully supported by conventional therapies [35]. Patients were also reported to revisit their decision to use CAM after the end of conventional treatment in order to regain strength and energy after going through chemotherapy or radiotherapy [36]. At the same time, fear of cancer recurrence or metastasis may also force patients to revisit their CAM decision and to try therapies which they rejected during the initial phase [37].

\section{Conclusion}

In summary the CAM decision making process is highly individualized, complicated and revolves around multiple factors. Patients' values, beliefs, knowledge and specific health care needs may affect this highly complex process.

\section{References}

1. World Health Organization (2013) Cancer.

2. Costa-Lotufo LV, Khan MT, Ather A, Wilke DV, Jimenez PC, et al. (2005) Studies of the anticancer potential of plants used in Bangladeshi folk medicine. J Ethnopharmacol 99: 21-30.

3. World Health Organization (2008) GLOBOCAN: cancer fact sheet.

4. Zollman C, Vickers A (1999) Users and practitioners of complementary medicine. BMJ, 319: 836-838.

5. World Health Organization (2008) Traditional Medicine.

6. National Center for Complementary and Alternative Medicine (2011) Expanding Horizons of Health Care. What is CAM?

7. Callahan LF, Wiley-Exley EK, Mielenz TJ, Brady TJ, Xiao C, et al. (2009) Use of complementary and alternative medicine among patients with arthritis. Prev Chronic Dis 6: A44.

8. Dhalla S, Chan KJ, Montaner JS, Hogg RS (2006) Complementary and alternative medicine use in British Columbia- a survey of HIV positive people on antiretroviral therapy. Complement Ther Clin Pract 12: 242-248.

9. Yeh GY, Davis RB, Phillips RS (2006) Use of complementary therapies in patients with cardiovascular disease. Am J Cardiol 98: 673-680.

10. Horneber M, Bueschel G, Dennert G, Less D, Ritter E, et al. (2012) How many cancer patients use complementary and alternative medicine a systematic review and meta-analysis. Integr Cancer Ther 11: 187-203.

11. Mao JJ, Farrar JT, Xie SX, Bowman MA, Armstrong K (2007) Use of complementary and alternative medicine and prayer among a national 
sample of cancer survivors compared to other populations without cancer. Complement Ther Med 15: 21-29.

12. Verhoef MJ, Balneaves LG, Boon HS, Vroegindewey A (2005) Reasons for and characteristics associated with complementary and alternative medicine use among adult cancer patients: A systematic review. Integr Cancer Ther 4: 274-286.

13. Ben-Arye E, Cassileth B, Heusser P, Afifi F, Saad B, et al. (2012) Complementary and integrative oncology in the cross-cultural region of the middle east and south asia. Evid Based Complement Alternat Med, 940961

14. Liu TG, Xiong SQ, Yan Y, Zhu H, Yi C (2012) Use of chinese herb medicine in cancer patients: A survey in southwestern china. Evid Based Complement Alternat Med, 769042.

15. Broom A, Adams J (2009) Oncology clinicians' accounts of discussing complementary and alternative medicine with their patients. Health, 13 317-336.

16. Shaharudin SH, Sulaiman S, Emran NA, Shahril MR, Hussain SN (2011) The use of complementary and alternative medicine among Malay breast cancer survivors. Altern Ther Health Med17: 50.

17. Puataweepong P, Sutheechet N, Ratanamongkol P (2012) A survey of complementary and alternative medicine use in cancer patients treated with radiotherapy in Thailand. Evid Based Complement Altern Med 670408.

18. Shih V, Chiang JYL, Chan A (2009) Complementary and alternative medicine (CAM) usage in Singaporean adult cancer patients. Ann Oncol 20: 752-757.

19. Malik IA, Khan NA, Khan W (2000) Use of unconventional methods of therapy by cancer patients in Pakistan. Eur J Epidemiol 16: 155-160.

20. Tarhan O, Alacacioglu A, Somali I, Sipahi H, Zencir M, et al. (2009) Complementary-alternative medicine among cancer patients in the western region of Turkey. J BUON 14: 265-269.

21. Taylor EJ (2005) Spiritual Complementary Therapies in Cancer Care. Semin Oncol Nurs 21: 159-163.

22. Robinson A, Mc Grail MR (2004). Disclosure of CAM use to medical practitioners: A review of qualitative and quantitative studies. Complement Ther Med 12: 90-98.

23. Gerber B, Scholz C, Reimer T, Briese V, Janni W (2006) Complementary and alternative therapeutic approaches in patients with early breast cancer: A systematic review. Breast Cancer Res Treat 95: 199-209.

24. Mao JJ, Palmer SC, Straton JB, Cronholm PF, Keddem S, et al. (2008) Cancer survivors with unmet needs were more likely to use complementary and alternative medicine. J Cancer Surviv 2: 116-124.
25. Verhoef MJ, White MA (2002) Factors in making the decision to forgo conventional cancer treatment. Cancer Practice, 10: 201-207.

26. Boon H, Brown JB, Gavin A, Stewart M (1999) Breast cancer survivors' perceptions of complementary/alternative medicine (CAM): making the decision to use or not to use. Qual Health Res 9: 639-653.

27. Hirai K, Komura K, Tokoro A, Kuromaru T, Ohshima A, et al. (2008) Psychological and behavioral mechanisms influencing the use of complementary and alternative medicine (CAM) in cancer patients. Annal Oncol 19: 49-55.

28. Hyodo I, Amano N, Eguchi K, Narabayashi M, Imanishi J, et al. (2005) Nationwide survey on complementary and alternative medicine in cancer patients in Japan. J Clin Oncol 23: 2645.

29. Balneaves LG, Bottorff JL, Hislop TG, Herbert C (2006) Levels of commitment: Exploring complementary therapy use by women with breast cancer. J Altern Complement Med, 12: 459-466.

30. Gupta M, Shafiq N, Kumari S, Pandhi P (2002) Patterns and perceptions of complementary and alternative medicine (CAM) among leukaemia patients visiting haematology clinic of a north Indian tertiary care hospital. Pharmacoepidemiol Drug Saf 11: 671-676.

31. Ezeome E, Anarado A (2007) Use of complementary and alternative medicine by cancer patients at the University of Nigeria Teaching Hospital, Enugu, Nigeria. BMC Complement Altern Med 7: 28.

32. Choi KS, Kim EJ, Lim JH, Kim SG, Lim MK, et al. (2007) Job loss and reemployment after a cancer diagnosis in Koreans-A prospective cohort study. Psychooncology 16: 205-213.

33. Bodeker G, Kronenberg F (2002) A public health agenda for traditional, complementary, and alternative medicine. Am J Public Health 92: 1582.

34. Shen J, Andersen R, Albert PS, Wenger N, Glaspy J, et al. (2002) Use of complementary/alternative therapies by women with advanced-stage breast cancer. BMC Complement Altern Med 2: 8.

35. Deng G, Cassileth BR (2005) Integrative oncology: Complementary therapies for pain, anxiety, and mood disturbance. CA: Can J Clin 55:109-116.

36. Balneaves LG, Weeks L, Seely D (2008) Patient decision-making about complementary and alternative medicine in cancer management: Context and process. Curr Oncol 15: s94.

37. Henderson JW, Donatelle RJ (2004) Complementary and alternative medicine use by women after completion of allopathic treatment for breast cancer. Altern Ther Health Med 10: 52-57. 